\title{
Observational Bias and the Clustering of Distant Eccentric Kuiper Belt Objects
}

\author{
Michael E. Brown \\ Division of Geological and Planetary Sciences, California Institute of Technology, Pasadena, CA 91125, USA; mbrown@ caltech.edu \\ Received 2017 May 12; revised 2017 June 13; accepted 2017 June 13; published 2017 July 21
}

\begin{abstract}
The hypothesis that a massive Planet Nine exists in the outer solar system on a distant eccentric orbit was inspired by observations showing that the objects with the most distant eccentric orbits in the Kuiper Belt have orbits that are physically aligned, that is, they are clustered in longitude of perihelion and have similar orbital planes. Questions have remained, however, about the effects of observational bias on these observations, particularly on the longitudes of perihelion. Specifically, distant eccentric Kuiper Belt objects (KBOs) tend to be faint and only observable near their perihelia, suggesting that the longitudes of perihelion of the known distant objects could be strongly biased by the limited number of locations in the sky where deep surveys have been carried out. We have developed a method to rigorously estimate the bias in longitude of perihelion for Kuiper Belt observations. We find that the probability that the 10 known KBOs with semimajor axis beyond 230 au are drawn from a population with uniform longitude of perihelion is $1.2 \%$. Combined with the observation that the orbital poles of these objects are also clustered, the overall probability of detecting these two independent clusterings in a randomly distributed sample is $0.025 \%$. While observational bias is clearly present in these observations, it is unlikely to explain the observed alignment of the distant eccentric KBOs.
\end{abstract}

Key words: catalogs - Kuiper belt: general

\section{Introduction}

Recently, Batygin \& Brown (2016, hereafter BB16) demonstrated that the most distant known objects in the Kuiper Belt have orbits that are physically aligned, that is, they are clustered in longitude of perihelion. Such an alignment is unexpected, because differential precession will destroy any such alignment on a timescale of 10-100 Myr. BB16 demonstrated that a distant giant planet in an eccentric orbit-referred to here as Planet Nine - could maintain an alignment for the age of the solar system if the longitude of perihelion of its eccentric orbit is oriented $180^{\circ}$ away from those of the clustered Kuiper Belt objects (KBOs). To date, no other viable explanation for a physical alignment of the orbits of distant eccentric KBOs has been proposed.

Previously, Trujillo \& Sheppard (2014, hereafter TS14) had noted that distant KBOs were clustered in argument of perihelion, $\omega$, a parameter that corresponds not to physical alignment but to a specific internal-orientation-with-respect-toitself of an orbit. While TS14 speculated that a distant planet might be responsible, no mechanism has been found for clustering $\omega$ of a population of KBOs by a planetary perturber without also having a physical clustering in longitude of perihelion. Madigan \& McCourt (2016, hereafter MM16) instead demonstrated that a massive disk of eccentric KBOs will generate an inclination instability that will naturally lead to clustering in $\omega$. To date, no other viable explanation for a clustering of $\omega$ (that does not also include clustering of longitude of perihelion) of distant eccentric KBOs has been proposed.

While the $\omega$ clustering reported by TS14 is robust and cannot be caused by any observational bias (see below), the clustering in longitude of perihelion reported by BB16 is certainly subject to observational bias. As a simple example, a magnitudelimited survey will preferentially find objects that are near their perihelion position, where they are brightest, and if such surveys are biased in the longitudes at which they are carried out, that bias will be reflected in the distribution of longitude of perihelion found. Given that biases in survey longitudes are known to exist (mainly, but not exclusively, caused by avoidance of the Galactic plane), the possibility of a bias in measured longitude of perihelion should be carefully considered. BB16 made a simple argument that the most distant eccentric KBOs should not be significantly more biased than slightly less distant eccentric KBOs-which show an essentially uniform distribution of longitude of perihelion-but it is clear that the bias toward finding objects at perihelion grows with eccentricity, so it is not obvious how applicable this simple argument is, particularly for the most eccentric orbits.

Because of this potential uncertainty about observational bias, it has been speculated that the clustering in longitude of perihelion might be purely an observational selection effect (Sheppard \& Trujillo 2016; Bannister et al. 2017; Lawler et al. 2017; Shankman et al. 2017). Assessing the impact of observational bias in longitude of perihelion is critical to understanding whether the observations point to a self-gravitating massive outer disk or to the presence of a giant ninth planet. Here we develop a rigorous method to estimate the bias in longitude of perihelion for distant eccentric KBOs. We apply the method to the distant eccentric KBOs originally identified by BB16 and to those that have been identified since to assess the possibility of the presence of Planet Nine.

\section{Significance of $\boldsymbol{\omega}$ Clustering}

Before discussing biases in the longitude of perihelion, we quickly discuss the argument of perihelion, $\omega$, and show how the observed clustering around $\omega \sim 0$ cannot be caused by observational bias, even though there are clear observational biases in $\omega$ for eccentric objects. In particular, eccentric objects with $\omega$ near 0 or $180^{\circ}$ come to perihelion and are thus brightest around the heavily observed ecliptic, so one would expect eccentric objects to be found preferentially around $\omega=0$ and $180^{\circ}$ even for a uniformly distributed population. At the 
moment of discovery, however, an object with $\omega=\omega^{\prime}$ and one with $\omega=180^{\circ}-\omega^{\prime}$ differ only in the direction of the ecliptic latitudinal component of their velocity vectors. As pointed out by $\mathrm{TS} 14$, there is no possible way to design a survey to be biased in favor of finding objects close to $\omega=0$ at the expense of objects with $\omega$ close to $180^{\circ}$ (or vice versa), yet the distant eccentric KBOs show this effect strongly.

While it is not possible to calculate the full observational selection bias of $\omega$, it is trivial to calculate the probability that objects would be exclusively clustered around $\omega=0$ or around $180^{\circ}$. In the original analysis, TS 14 found that the 12 most distant eccentric KBOs-those with semimajor axis 150 au and greater-cluster within $43^{\circ}$ of $\omega=0$. The probability that 12 such objects would cluster around either 0 or $180^{\circ}$ is simply $2 \times 2^{-12}$, or $0.04 \%$. Note that here and throughout this paper we refer to KBOs as all multi-opposition solar system objects with perihelion distance beyond Neptune's orbit.

Since the original work of TS14, nine new KBOs with semimajor axis 150 au or greater have been found. Of these, seven are closer to $\omega=0$ than to $\omega=180^{\circ}$. The probability that 19 or more of 21 objects would be so clustered is $2 \times 2^{-21} \times[C(21,2)+C(21,1)+1]$ where $C(n, m)$ is the number of independent combinations of $m$ objects from a population of $n$. The probability of this occurrence is thus just $0.022 \%$.

No sophisticated debiasing needs to be done to show that the clustering in $\omega$ is highly significant. This strong signalunexplained by the mechanism proposed by TS14-led MM16 to the realization that this clustering could be caused by a massive distant disk causing an inclination instability in the outer solar system. A distant eccentric Planet Nine, in contrast, clusters longitude of perihelion and pole position, rather than $\omega$. A population of orbits aligned in longitude of perihelion with poles clustered around a position offset from the ecliptic pole will generally, but not exclusively, also have clustered $\omega$. Unfortunately no simple calculation gives pole position bias, so we continue to use the clustering in $\omega$ as an imperfect statistical proxy for clustering in pole position.

Clustering of distant eccentric KBOs in $\omega$ (or, alternatively, in pole position) is firmly established. To date, the only viable explanation for this clustering is either the proposal of inclination instability by MM16 or the proposal of Planet Nine by BB16. These proposals differ most in their predicted distribution of longitude of perihelion. The inclination instability shows no preference for clustering in longitude of perihelion, while Planet Nine confines the longitudes.

If in fact there is no clustering in longitude of perihelion, the robust clustering in $\omega$ is currently only explicable by the presence of a massive outer disk of material inducing an inclination instability through self-gravity, as proposed by MM16. If, on the other hand, the clustering in longitude of perihelion is a true effect, rather than an apparent one caused by observational bias, Planet Nine remains the only currently proposed explanation. We now examine observational biases in longitude of perihelion to determine which of these hypotheses appears more likely.

\section{Observational Bias in Longitude of Perihelion}

The best method for determining the effects of observational bias on the known KBOs would be to have complete information from all of the surveys conducted to date, including their depth, precise coverage, and efficiency. Such information is unknown for the majority of the surveys that led to the discoveries of the cataloged KBOs. In many cases, nothing is published about the discovery survey; the existence of the object is simply cataloged by the IAU Minor Planet Center (see http://www.minorplanetcenter.net/iau/Unusual. html). The cataloged information is sufficient to determine the ecliptic longitude, ecliptic latitude, heliocentric distance, and brightness of every object at the time of its discovery.

We develop a novel method to use the circumstances of discovery of the ensemble of all KBOs in the catalog to rigorously estimate the statistical distribution of longitudes of perihelion expected for distant eccentric KBOs. Conceptually, the method relies on the idea that each KBO discovery can be thought of as a survey that could have discovered a distant eccentric KBO had that object been bright enough and in the same place. We proceed as follows: for each distant eccentric KBO (a "parent object") we construct a synthetic population of new objects assuming an identical absolute magnitude and identical orbital elements for a uniformly selected longitude of perihelion and mean anomaly (in practice we also assume symmetry across the ecliptic plane, so our constructed population also includes orbits where we replace $\omega$ with $-\omega$ ). We tally the ecliptic longitude, ecliptic latitude, heliocentric distance, and expected magnitude of each object in the synthetic population. We call this the "uniform population" of the parent KBO. Next, for every KBO discovery in the catalog we assess whether or not one member of the uniform population of the parent object exists at the ecliptic longitude and latitude of the observation and whether that member is brighter than the actual detected $\mathrm{KBO}$. If so, we know that a survey was being undertaken at that point that could have detected one of the uniform population. (In practice, we look for members of the uniform population within $1^{\circ}$ of the discovery location of the KBO.) Finally, we tabulate the longitudes of perihelion of the members of the uniform population that could have been detected at that discovery location. We now know that the survey that resulted in that particular KBO discovery was sensitive to members of our uniform population if they had had a particular longitude of perihelion. This procedure is repeated for every cataloged $\mathrm{KBO}$ discovery to determine the probability distribution function of the longitude of perihelion of the parent $\mathrm{KBO}$ assuming that the population is uniformly distributed in longitude of perihelion and mean anomaly.

A concrete example makes this procedure more clear. Consider 2013 RF98, the most eccentric of the objects originally identified by BB16, as the parent object of a uniform population. Next, consider the discovery of a randomly cataloged KBO, 2015 GP50, which, at the moment of discovery, had an ecliptic latitude of -11.2 and a magnitude of 24.8. Examining the orbit of 2013 RF98, we find that it crosses $-11^{\circ} .2$ twice, once $27^{\circ}$ from perihelion, when it has a magnitude of 24.6, and once closer to aphelion, when it has a magnitude of 28.7. Near aphelion, the uniform population would not have been detectable at this latitude, but at its magnitude closer to perihelion it could have been detected by the observation that discovered 2015 GP50. The KBO 2015 GP50 was discovered at a longitude of $196^{\circ}$, thus the member of the uniform population that is detectable has a longitude of perihelion of $196^{\circ}-27^{\circ}=169^{\circ}$. This specific observation is thus biased to finding this specific longitude of perihelion for 
this specific parent object. If we now consider not a single KBO discovery but all KBO discoveries, we find a statistical distribution of the longitudes of perihelion in which discoveries of the uniform population of 2013 RF98 could have been made. We thus create a separate statistical distribution of expected longitudes of perihelion for each distant eccentric KBO.

This conceptual framework relies on the assumption that $\mathrm{KBO}$ discoveries roughly represent the coverage and depth of the combined surveys. This assumption is clearly false for the latitude distribution, where more KBOs are discovered at low ecliptic latitudes simply because of their greater numbers. We correct this bias by scaling by expected density of KBOs at a given latitude. To approximate this expected density we use the method developed by Brown (2001) to determine the inclination distribution and convert it to a latitudinal distribution assuming circular orbits. The final results are not sensitive to the precise latitudinal distribution chosen.

A second way in which the assumption that $\mathrm{KBO}$ discoveries are uniform with search area is violated is in the known longitudinal bias in the discovery of resonant KBOs, which are over-discovered near their perihelion positions, which are related to the position of Neptune. The easiest way to avoid this problem is to discard all discoveries of Plutinos, which are, by far, the most numerically prominent and most spatially correlated of the resonant objects. In practice we simply discard all discovered objects with semimajor axes below 40 au. This constraint also forces us to retain only multiopposition KBOs with orbits known accurately enough to calculate this parameter. We note, however, that relaxing this assumption makes the final results of this analysis more significant. Nonetheless, we conservatively retain this constraint.

One other important assumption is that a distant eccentric KBO could always be discovered if a closer but fainter KBO was discovered at its predicted location. This assumption can be violated if the discovery survey is not sensitive to distant objects. Some of the nearest KBOs and many Centaurs, for example, have been discovered in surveys searching for nearEarth objects, which do not have observational baselines sufficiently long to be sensitive to more slowly moving distant objects. To exclude these surveys we will consider only discoveries of objects at distances greater than 30 au. Even for normal KBO surveys, some of the distant eccentric KBOs might not be detectable due to their low apparent speed even though they are still brighter than the magnitude limit. Sedna, for example, would be visible to a distance of 225 au to a survey that went to a depth of 25th mag, but few surveys are sensitive to the slow motions of such distant objects. We will thus place an upper bound of 90 au on the most distant object that any survey could see. This value is probably a conservative estimate and will have the effect of making biases in longitude of perihelion stronger than they might be in real life. In total, we use the observations of 1248 objects to determine our expected distributions.

Using all of these constraints, we calculate expected statistical distributions of longitude of perihelion for each of the 10 known KBOs with semimajor axis beyond 230 au. These include the six originally identified by BB 16 and the four that have been discovered since that time (Figure 1). The expected distributions of longitude of perihelion are highly structured and highly individual. One trend is easily seen. The brightest objects (Sedna, 2007 TG422) are among the most

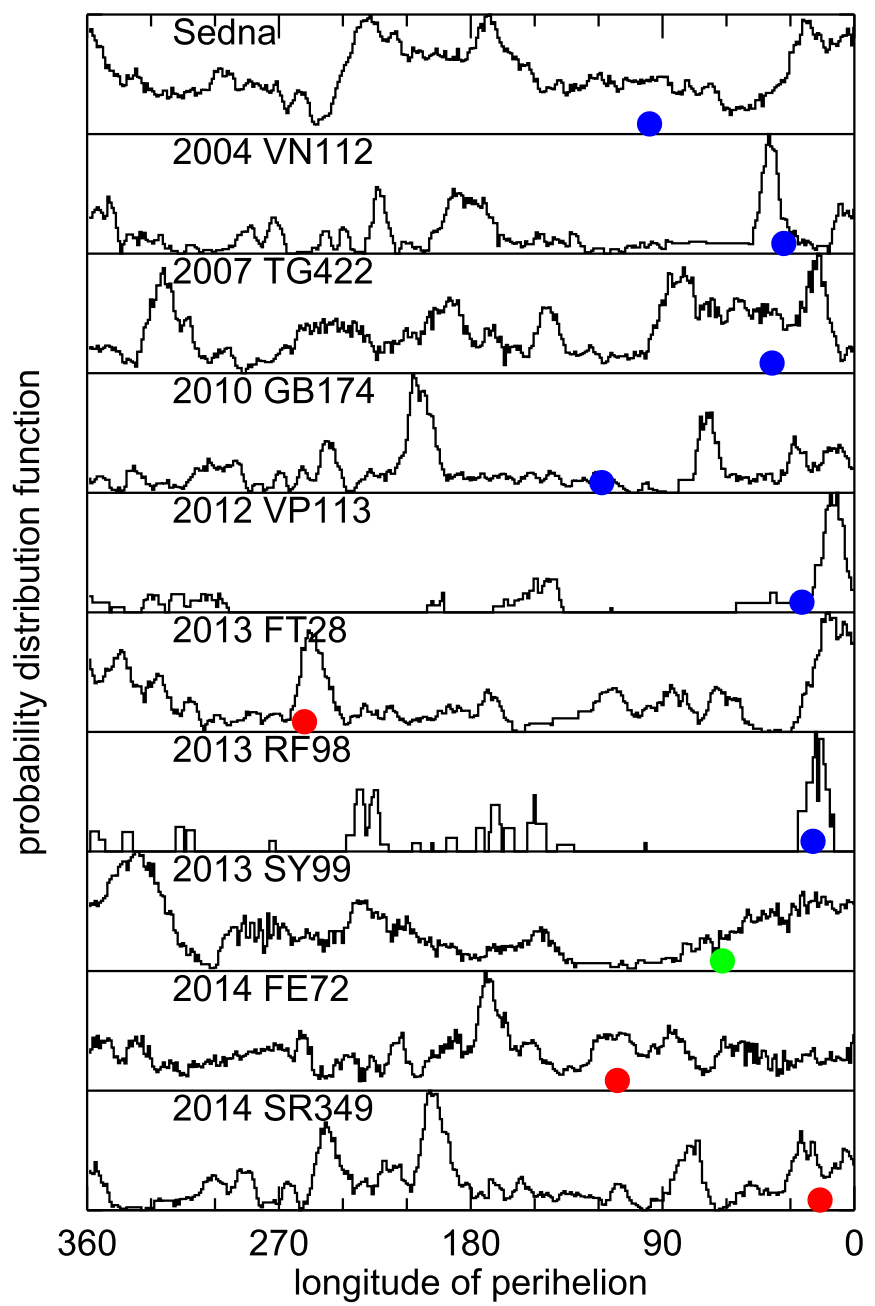

Figure 1. Calculated probability distribution functions for the expected distribution of longitude of perihelion assuming a population of objects with identical orbital elements but uniformly distributed in longitude of perihelion and mean anomaly. The colored dot shows the actual longitude of perihelion of each object. The blue dots note the six KBOs originally discussed by BB16, the red dots show the newer discoveries of Sheppard \& Trujillo (2016), while the green dot shows the discovery of Bannister et al. (2017). While observational biases are strong in the expected distribution of longitude of perihelion, it is clear that for nearly all objects the bias toward discovering the object with its actual longitude of perihelion is not severe.

uniform in their expected discovery distributions. Many surveys could have found the very bright Sedna even quite far from its perihelion, for example. The structure seen in the remaining objects is only understandable after analysis. The distributions of 2012 VP113 and 2013 RF98, for example, are the most nonuniform. These two objects both come to perihelion at high ecliptic latitude, so their populations are primarily observable at high latitude, yet few surveys reach the required depth at these latitudes. The longitudes of perihelion of these objects are highly biased by the limited number of surveys that could have detected such a population. The structures in the other distributions are similarly functions of latitude of perihelion, brightness, and the distribution of surveys in the sky.

\section{Comparison to Observations}

We now see that measurement of the longitude of perihelion for a distant eccentric population is highly biased by the 


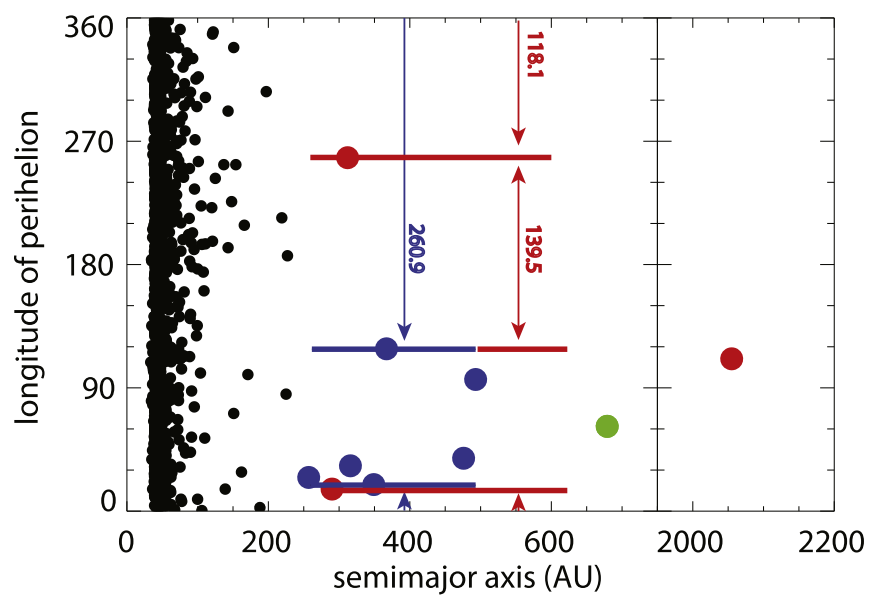

Figure 2. Longitude of perihelion of KBOs as a function of semimajor axis (note the change in axis to include 2014 FE72 with a semimajor axis of 2055 au). The blue objects are the original six discussed by BB16, which included all KBOs with semimajor axes greater than 230 au known at the time. The red points are subsequent discoveries from Sheppard \& Trujillo (2016) while the single green point is from Bannister et al. (2017). Angular distances between subsets of KBOs discussed in the text are noted.

specific area of the sky and depth of individual surveys, as expected. With our determination of this bias, we can now examine whether the discoveries of distant eccentric KBOs are consistent with being selected from a distribution that is uniform in longitude of perihelion or if, indeed, they are clustered.

We first consider the six distant KBOs that BB16 reported as clustered in longitude of perihelion: Sedna, 2004 VN112, 2007 TG422, 2010 GB174, 2012 VP113, and 2013 RF98. At the time, these were all of the known KBOs with semimajor axis beyond $230 \mathrm{au}$. To understand the statistically expected distribution of longitudes of perihelion for these bodies assuming a uniformly distributed population, we perform 100,000 population samplings in which we create a new selection of six detected KBOs by randomly choosing a longitude of perihelion from the expected probability density function for each of the six objects. We then examine the statistics of these 100,000 realizations.

The longitudes of perihelion of the six real objects are distributed such that the maximum angle between any pair of angularly adjacent objects is $260^{\circ} .9$ (Figure 2). For the 100,000 realizations of this population assuming a uniform distribution in longitude of perihelion, the maximum angle between two angularly adjacent objects is $260^{\circ} .9$ or higher in only 1437 cases. If the longitudes of perihelia of distant eccentric objects are uniformly distributed, we would expect a longitude clustering as tight as the one observed only $1.4 \%$ of the time. We also compute the Rayleigh $z$ statistic of the data and the random sample and find that the Rayleigh $z$ value of 0.80 of the data is exceeded only $2.0 \%$ of the time in the random data. In contrast, the simple estimate from BB16 (which also took into account the clustering in latitude of perihelion, which is ignored here) suggested that the clustering should be observed only $0.7 \%$ of the time. We regard the rough agreement from independent ways of estimating the significance of this result as encouraging.

With only the six objects defined here, the probability of $\omega$ clustering becomes $2 \times 2^{-6}$ or $3.1 \%$. Restating these two findings, we see that there is only a $3.1 \%$ chance that the values for $\omega$ are equally distributed about 0 and $180^{\circ}$, suggesting that some mechanism is clustering the distant KBOs in $\omega$. We likewise find that there is only a $1.4 \%$ chance that the longitudes of perihelia are distributed uniformly, suggesting that these values are likewise clustered. The combined probability that both of these clusters would be found in random data is thus $0.043 \%$.

Since the original analysis of BB16, Brown \& Batygin (2016) demonstrated that in the Planet Nine hypothesis, in addition to a cluster of distant eccentric KBOs in longitudes of perihelion opposite to that of Planet Nine (the "anti-aligned population"), there should also exist a smaller population of objects with longitudes of perihelion aligned with Planet Nine (the "aligned population"). Four new distant eccentric KBOs (with semimajor axis greater than $230 \mathrm{au}$ ) have been discovered since the initial analysis (Sheppard \& Trujillo 2016; Bannister et al. 2017). Of these, three fit well with the anti-aligned population (2013 FT28, 2014 FE72, and 2013 SY99), while one is consistent with being the first recognized member of the aligned population (2014 SR349). The realization that we now expect two separate, oppositely oriented populations requires a different metric for assessing the match between the expected and observed populations. Instead of examining the largest angle between the longitudes of perihelion of any two angularly adjacent objects, we look at the second largest angle between any two angularly adjacent objects. In a population with two well separated, oppositely oriented groups, this second largest angle will be maximized. In practice, we would have also considered the observed population of objects to be clustered if there had been only a single cluster rather than two. Such a cluster would have a large largest angle but a small second largest angle. To overcome this problem we take either the second largest angle or half of the largest angle, effectively mimicking the effects of two populations even if only one is observed. In the real population of 10 distant eccentric KBOs, the separation in longitude of perihelion between the antialigned group and 2013 SR349 is $139^{\circ} .5$ in one direction and $118^{\circ} .1$ in the other direction. Our second largest angle is thus $118^{\circ} .1$. We again perform 100,000 random iterations and compare this expected population to the real observations. In only 1201 cases is the second largest separation between longitudes of perihelion as large as or larger than $118^{\circ} .1$. The probability that the distant eccentric KBOs would be distributed in longitude of perihelion as extremely as the observations are if the underlying distribution were uniform is $1.0 \%$. The calculated Rayleigh $z$ statistic of this population (which is only sensitive to a unimodal distribution) with a value of 0.62 is exceeded in $2.2 \%$ of the random sample. While the discovery of four new distant eccentric KBOs might have been expected to increase our confidence in these populations significantly, the realization that we are observing two opposing populations rather than one single clustered population necessarily dilutes the signal.

One of these objects (2014 FE72) has $\omega=134^{\circ}$, while the rest are clustered about zero. Sheppard \& Trujillo (2016) use this fact to exclude 2014 FE72 from consideration as part of the clustered population, but show that such objects are indeed expected in the Planet Nine hypothesis. They are simply precessing around a displaced pole but have temporarily circulated past the north ecliptic pole. Ecliptic-based Keplerian orbital elements are a poor descriptor of the orbits in this case. Nonetheless, we continue to use the simple measure of $\omega$ as a proxy for pole clustering and find that if the true values of $\omega$ are 
uniform about 0 and $180^{\circ}$, the probability that nine or more of 10 values for $\omega$ would be clustered about either is $2.1 \%$. The combined probability of both of these parameters being clustered is $0.025 \%$.

The original $\omega$ clustering discussed by TS14 that led to the MM16 hypothesis of inclination instability includes all KBOs with semimajor axis 150 au and larger. To date, 21 such KBOs are known, and 19 have $\omega$ closer to 0 than to $180^{\circ}$. The probability of this clustering occurring randomly is a mere $0.022 \%$. Examination of the longitude of perihelion (Figure 2) shows that this parameter, too, has some structure down to a semimajor axis of $150 \mathrm{au}$, but it is clear that the clustering in longitude of perihelion is beginning to break down. This behavior was seen in the population simulations of, where it was noted that the longitude of perihelion changed from being highly clustered at large semimajor axis, to moderately to notat-all clustered as semimajor axis decreased. We thus do not expect the objects clustered in longitude of perihelion beyond $150 \mathrm{au}$ to be as significant as those beyond $230 \mathrm{au}$ that we initially considered. We nonetheless assess the observational biases. Once again performing 100,000 iterations of a population uniformly distributed in longitude of perihelion, we find that 43,590 have a second largest angle of longitude of perihelion between two KBOs as large as or larger than $48^{\circ} .2$, the value seen in the real data (while 39,753 have a largest angle of $67^{\circ} .9$ or greater, like the data). The Rayleigh $z$ test likewise shows no significance to this clustering. In short, for objects with semimajor axis $150 \mathrm{au}$ and larger there is no statistically significant clustering of longitude of perihelion into one or two groups, yet the cluster of $\omega$ is highly significant. This discrepancy shows, we believe, the expected blending of the longitudinally clustered objects with large semimajor axis into the background population with smaller semimajor axis, and the uncertainty as to where precisely to draw the line between distant objects that are affected by Planet Nine and those that are not. At greater semimajor axes, the clustering in longitude of perihelion is robust as expected.

\section{Conclusions}

We have shown that measurement of the longitude of perihelion of a population of distant eccentric KBOs is subject to considerable observational bias, but that this bias is unlikely to be responsible for the observed clustering in longitude of perihelion. If distant eccentric KBOs were uniformly distributed in longitude of perihelion, observations of the original six objects with semimajor axis beyond 230 au that led BB16 to suggest the existence of Planet Nine would only find the extreme clustering observed $1.4 \%$ of the time. Including the four most recently discovered KBOs with semimajor axes beyond 230 au lowers that probability to $1.2 \%$.

Clustering in $\omega$ of distant eccentric KBOs is firmly established. Thus determination of the veracity of the clustering in longitude of perihelion is critical to understanding the gravitational forces sculpting the outer solar system. With no clustering in longitude of perihelion, the only currently proposed viable mechanism for causing $\omega$ clustering is the suggestion by MM16 of a massive outer disk causing an inclination instability and $\omega$ clustering. If, on the other hand, longitude of perihelion and pole position (which roughly manifests itself as $\omega$ for an offset pole) are clustered, then Planet Nine is the only currently proposed viable hypothesis. By rigorously estimating the effects of observational bias, we have shown here that the Planet Nine hypothesis is by far the more likely of these scenarios. The probability that the combination of the alignment of the longitudes of perihelion with the clustering in pole position (using the $\omega$ proxy) that is seen in the KBOs with semimajor axes beyond 230 au would occur by chance in a uniformly distributed population is only $0.025 \%$. While explanations other than Planet Nine might one day be found to explain these observations, the significance of the observations themselves appears secure.

We would like to thank Ann-Marie Madigan for the discussion that inspired this analysis and Elizabeth Bailey, Konstantin Batygin, and Ian Wong for critical readings of the manuscript.

\section{References}

Bannister, M. T., Shankman, C., Volk, K., et al. 2017, AJ, 153, 262 Batygin, K., \& Brown, M. E. 2016, AJ, 151, 22 Brown, M. E. 2001, AJ, 121, 2804

Brown, M. E., \& Batygin, K. 2016, ApJL, 824, L23

Lawler, S. M., Shankman, C., Kaib, N., et al. 2017, AJ, 153, 33

Madigan, A.-M., \& McCourt, M. 2016, MNRAS, 457, L89

Shankman, C., Kavelaars, J. J., Lawler, S. M., Gladman, B. J., \& Bannister, M. T. 2017, AJ, 153, 63

Sheppard, S. S., \& Trujillo, C. 2016, AJ, 152, 221

Trujillo, C. A., \& Sheppard, S. S. 2014, Natur, 507, 471 